\title{
THEORY AND APPLICATION OF CERENKOV COUNTING
}

\author{
H. H. Ross \\ Analytical Chemistry Division \\ Oak Fidrye National Laboratory* \\ Oak Ridge, Tennessee 37330 (USA)
}

\begin{abstract}
The production of Cerenkov radiation by charged particles moving through a transparent medium (the Cerenkov generator) is a strictly physical process that is virtuaily independent of the chemistry of the medium. Thus, it is possible to calculate easily all of the important parameters of the Cerenkov process such as excitation threshold, eritission intensity, spectral distribution, directional characteristics, and time response. The results of many of these physical effects are unknown in conventional liquid scintiliation spectroscopy and, therefore, many unique assay techniques have been developed as a result of their consideration. This paper will discuss the theoretica? basis of the Cerenkov process, explore the unusua? characteristics of the phenomenon, and demonstrate how these unusual characteristics can be used to develop equally unusual counting methodologies. Particular emphasis will be directed toward an analysis of published data (obtained with convantional liquid scintillation instrumentation) that would be difficult or impossible to obtain with other counting techniques. Also discussed is a new type of sample vial that uses an isolated waveshifting system. The device was specifically desigied for Cerenkov counti.ug.
\end{abstract}

*Operated by the Union Carbide Corporation for the Energy Research and Development Administration.

By acceptance of this article, the publisher or recipient acknowledges the U.S. Government's right to retain a nonexclusive, royalty-free license in and to any copyright covering the article.

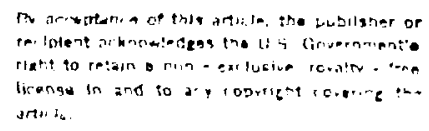


Admiring, in the gloomy shade,

Those little drops of light.

Edmund Waller

\section{INTRODUCTION}

Although the concept of counting a radionuclide dissolved in a liquid via Cerenkov radiation was demonstrated by Belcher (1) over 20 years ago, it is only within the last five years that a significant number of real applications have appeared in the open literature. This lack of interest was most probably due to the difficulty in measuring the very feeble emission generated by beta particles in the range of 0.5 to $2.0 \mathrm{MeV}$. For example, a $1.0 \mathrm{MeV}$ beta particle in water dissipates only about $1 \mathrm{KeV}$ of energy in the 300 to $700 \mathrm{~nm}$ spectral region (perhaps $30-45$ photons). Add to this tenuous photon emission the limited spectral sensitivity and conversion efficiency of early photomultiplier tubes, and the experimental difficulties become obvious. 0ther problems, such as dark current $(S / N)$, directional considerations, and noisy electronics only compounded the dilemma. However, new instrumentation concepts and components for liquid scilitillation counting have changed the situation significantly; high sensitivity Cerenkov counting has become a routine operation in many 1aboratories.

Jelley (2) has written an excellent account of the theory and application of Cerenkov radiation. The major application and instrumentation emphasis is, however, directed toward problem areas in high energy physics. More recent discussions (3-5) cover the general area of using commercially available liquid scintillation spectrometers for the assay of disso?ved nuclides in sma 11, conventional samples. These papers describe a wide range of experimental conditions that include typical detection efficiencies, instriment set-up, quenching effects, threshold energy response, sample yolume, and directional considerations. A11 of these papers emphasize three important considerations peculiar to Cerenkov counting - energy discrimination, chernical quenching, and the absence of conventional scinisillation fluors in the system. A view of these special characteristics reveals the unique application areas of Cerenkov spect.roscopy. 


\section{Energy discrimination}

Perhaps the most unusual aspect of Cerenkov counting is the presence of an energy threshold effect that is solely a function of the refractive index of the Cerenkov generator solid, liquid, or gas. The result of this effect is that particles below the energy threshold create no photon emission; particles above the threshold do generate a photon emissior which becomes greater as the initial energy of the particle becomes further separated from the threshold energy. The threshold energy for electrons (betas) in any medium can be approximated using Equation 1:

$$
\begin{aligned}
& E_{T}=511\left(\frac{1}{\sqrt{1-\frac{1}{n^{2}}}}-1\right) \\
& \text { where } E_{T}=\text { threshold energy (KeV) } \\
& n=\text { refractive index }
\end{aligned}
$$

The actual threshold energy cannot be calculated exactly using Equation 7 because, in real systems, the change of refractive index as a function of wavelength must be considered along with the spectral sensitivity of the experimental photon detector. However, one sees in Table I that the electron energy threshold varies over a rather narrow range (using $n_{p}$ ) for a considerable change in refractive index. Thlus, for practical purposes, $E_{0}$ at $n_{D}$ is fair?y realistic. But, it should be noted that sone materials exhibit significant changes in refractive index in spectral regions (UV) where phototubes are sensitive. In these cases, major shifts can be observed in the energy threshold (ó).

One of the best examples of an application that takes advantage of the energy threshold effect is a demonstration of the rapid determination of phosphorus-32/33 mixtures by Brown (7). Analysis of these mixtures by liquid scintillation couriting does not offer a sufficiently high degree of sensitivity or accuracy because of the relatively low energy resolution inherent with this method. However, in an aqueous Cerenkov system, fhosphorus-33 lies below the energy threshold and thus yields no response at all; phosphorus-32, which lies well above the threshold gives a 
Table ?

CERENKOV ENERGY THRESHOLD FOR ELECTRONS IN VARIOUS SOLVENTS

\begin{tabular}{lcc}
\multicolumn{1}{c}{ Solvent } & : & Threshold (KeV) \\
ethyl trifluoroacetate & 1.31 & 283 \\
water & 1.33 & 263 \\
ethyl alcohol & 1.36 & 243 \\
40\% sucrose & 1.40 & 219 \\
toluene, 84\% sucrose & 1.50 & 174 \\
bromoform & 1.60 & 145 \\
methylene iodide & 1.76 & 111
\end{tabular}

strong reaction. Any counts observed in a phosphorus $-32 / 33$ mixture must be due only to the -32 contribution. (The response of -33 was experimental1y determined to be $<0.01 \%)$. By combining a Cerenkov count with a liquid scintillator count, Brown was able to determine mixtures of the phosphorus isotopes over an extremely wide range with errors usually less than 1.5\%. The absolute accuracy of this method is limited mainly by the degree of uncertainty of phosphorus-33 liquid scintillation and the phosphorus -32 Cerenkov counting efficiency determinations.

The above example must be considered to be a "classic" since only one nuclide in the mixture gives any Cerenkov response while the other is completely masked. Nevertheless, many practical measurement systems have been developed where two (or more) nuclides have been assayed by taking advantage of the low detection efficiency of nuclides whose maximum beta energy lies above the threshold, but not significantity above. For exar:? Te, thulium-170 with a beta energy maximum of almost 1 MeV counts with only about 5-6\% efficiency. Using similar techniques, Randolph (8) has determined mixtures of Sr-89/90; Buchtela and Tschurlovits (9), $\mathrm{Sr}-89 / 90$ and $\mathrm{Y}-90$; and Ross (10), mixtures of $\mathrm{Cl}-36$ and $P-32$. In each of these examp les, some Cerenkov $r$ asponse was observed from every nuclide. The differences wera sufficiently different, however, so ihat errors generinly in the range of $5 \%$ were obtained over tine span of mixture 
concentrations that were studied. The energy threshold technique is clearly not strictly limited to the theoretical cut-off value.

Chemical quenching

If any experimental parameter could be said to play a pivotal role in the success or failure of 1 iquid scintillation counting, it must certainly be that of color and chamical quenching. The quenching phenomenon must. be considered in virtually every aspect of the sample measurement - selection of cocktail formulation, sample preparation, instrument settings and correction procedures, etc. In almost one half of the liquid scintillation papers published in the last two years, a quenching problem and its solution was a major aspect of the investigation. In Cerenkov counting the situation is somewhat different; there is no known mechanism for chemical quenching to occur it does not exist. This simple fact has virtually revolutionized the techniques used for sampie preparation. Powerful chemical treatments that would be precluded frcm use with scintillation samples can be applied freely in Cerenkor systems. Dissolutions in strong acids, rapid molten salt fusions, and use of strong oxidants are all acceptable techniques. This new freedom for sample selection and processing has made a large impaci on the recent growth of Cerenkov counting applications.

Although it is still true that color quenching effects can exist (and are in fact quite important), the ability to use strong chemical methods to treat samples can often completely eliminate color problems. A recent publication (11) describes the destruction of blood platelets by treatment with $0.5 \mathrm{~N}$ sodium hydroxide and a subsequent direct count for P-32. The authors report that although the absolute counting efficiency is reduced from that obtained with scintillators, the advantages of a ieduction in time, material, and expense are realized. They also note that the ease of sample recovery from the counting solution commends the approach to studies requiring subsequent analyses of the platelet lysate. Other recent examples of specific sample treatment include plant extracts and parts $(12,13)$, gels $(12)$, and biologica! materials in general (17).

Although forceful handing of samples to remove color quenchirg is possible, some investigators opt for a quench 
correction technique when the quenching is not severe. Virtually all of the usual correction methods have been examined and, contrary to a report by Elrick and Parker (15), the channels ratio may be amor:g the best $(16,17,18,19)$. An alternate solution that has been proposed to circumvent color problems is the use of a "Cerenkov insert" as described by Ballance and Johnson $(20,21)$. Here, the sample is contained in a smal1, opaque container and dipped into the Cerenkov genertiting medium. Energetic particles pass through the container and into the solution that creates the Cerenkov emission. Since the colored sample is not dissolved or dispersed in solution, color effects are avoided. However, here it must be asked what this technique actually accomplishes. The sample, which is a seif-contained entity, is no longer free of self absorption effects. The result is that reduced counting efficiencies are observed when compared to homogeneous counting. The sinall size of the insert also obviates against another benefit of Cerenkov liethods, the use of large samples for lowered detection limits. It would appear that any advantage gained by use of the insert could aiso be realized by substituting a conventional liquid scintillator for the Cerenkov medium, with a considerable enhancement of the detection efficiency. Thus it is difficult to discern the application area of this method.

Spectral distribution, füuors, and counting efficiency

Since Cerenkov emission is solely the result of a rapid de-acceleration of a charged particle in a transparent medium, the use of conventional scintillation fluors is not necessary. This is the essential roason that chemical quenching does not occur in pure Cerenkov systems - there is simply nothing to chemicaliy quench. The theoretical spectral output from a transparent medium ranges between its spectral transmission linits over the range where the refractive index is greater than one. The relative spectral yield varies as $1 / \lambda^{3} d \lambda$ and is the reason that the familiar Cerenkov giow in swimming pool reactors always appears blue to the eye. Wider range detectors show that even greater emission occurs in the ultraviolet speciral region as predicted by theory.

The large majority of phototubes used for scintillation counting exhibit a significant drop in sensitivity in the ultraviolet region and, thus, much of the energy from a 
given particle event is not utilized for detection. For high-energy particles, this is not a significant probien; sufficient visible (and near UV) photons are generated to reliably trigger the phototube and counting circuits. However, for particles onily marginally above the energy threshold, there is not a sufficient number of detectable photons and the observed counting efficiency is reduced proportionately. It appeared obvious to many investigators that the use of a wavelength shifter in the Cerenkov medium could be used to recover and use much of the wasted ultraviolet radiation. The use of such a material would also destroy the directional characteristic of the emission and further enhance the detection efficiency (22).

Eerly work by Herberg and Marshall (23) demonstrated two materials that gave a significant gain in water. The better of the two, 2-amino-6, 8-naphthalenedisulfonic acid, gave an increase in pulse height of $30 \%$. This compound has been widely used since its utility was first demonstrated. Another significant increase in pulse height (as much as 100\%) was shown to result from the use of 4 -meth. lumbelliferone by Porter (24). A large number of other water soluble compounds have been proposed with various levels of success; many of these materials were examined in detail with regard to efficiency gain, stability, and other experimental parameters (22).

An important comment must be made at this point. Aithough the use of a wavelength shifting compound can result in a significsint increase in counting efficiency in idea? systems, in practice, one must exercise a great deal of care when they are used. The reason for this is that once such materials are added to the Cerenkov generator, the system becomes a hybrid Cerenkov-scintillation medium. As a result, many of ihe advantages described earlier cannot be attained. The most important factor is the realization that chemical quenching of the waveshifter can occur. Thus, much of the frestom in sample pre-treatment is lost and, along with it, the convenience of the "pure" Cerenkov methoc. Is there a way to "... have your cake and eat it too?" The answer is yes.

\section{MATERIALS B.ND METHODS}

Baillon, et.al., (25) have demonstrated that an increase by about a factor of 3.1 in photoelectron omission from Cerenkov light can be obtained by coating a standard 
photomultiplier tube with a thin deposit of a wavelength shifter (lithium fluoride). Although the coating operation is straightforward and requires only simple manipulation, it does not appear to be a feasible approach for a general purpose liquid scintillation counting system. This work, however, suggested the design of a new type of counting vial specifically for Cerenkov measurements using a waveshifter. The design of the vial is shown in Figure 1. The device consists of two concentric chambers. The outer chamber is used to contain a wavelength shifting solution that may be either organic or inorganic. In this work, the opening to the chamber was fitted with a stopper so that various waveshifting solutions could be tested. The inner chamber is used to hold the counting sample - about $10 \mathrm{ml}$ maximum. The entire assembly is made of quartz so that efficient transfer of the ultraviolet radiation from the inner chamber to the shifter can take place. Since the quartz vial is definately not a throw-away item, the inner chamber of the vial is lined with a thin (fy 1.8 mil) polyethylene bag. The use of this liner prevents radioactive contamination of the internal chamber surface yet permits a high degree of UV transmission. The construction of a similar all polyethylene vial appears feasibie but this fabrication was not attempted.

Scintillators and other materials used in this study were scintillator or reagent grade. The strontium- 89 radionuclide was prepared by irradiation of enriched strontium-88 nitrate (98\% enrichment) in the Oak Ridge Research Reactor. The initial standardization of the tracer was carried out by $4 \pi \beta$ counting techniques. A Packard series 3000 liquid scintillation counter was used for the Cerenkov measurements. Maximum amplifier gain and wide window settings were used.

\section{RESULTS}

The strontium-89 nitrate tracer was dissolved in $0.1 \mathrm{~N}$ $\mathrm{HCl}$. An aliquot of this solution was added to $10 \mathrm{ml}$ of $0.01 \mathrm{~N} \mathrm{HC}$ ? and this sample was placed into the inner chamber of the vial and sealed with a screw cap. The aliquot was selected so that about 30,000 dpm were contained in the sainple. The outer chamber wis filled with various soiutions with and without an added waveshifter. A count of the sample was made so that a total of at least 50,000 counts were coilected in each test. The relative counting efficiency 
was determined for each counting combiriation; the results are summerized in Table II.

\section{Table II}

RELATIVE COUNTING EFFICIENCY OF Sr-89

USING AN ISOLATED WAVELENGTH SHIFTING VIAL

Waveshifter

Chambe: Contents

Concentration

Relative

water

$100 \%$

1.00

ethanol

95\%

1.02

benzene

$100 \%$

1.06

toluene

$100 \%$

1.07

dimethyl POPOP

$0.7 \mathrm{gm} / 1$

1.88

4-methyl-

in toluene

umbell iferone

$0.5 \mathrm{gm} / 1$

1.69

2-naphthol-3,

6-disulfonic

acid-sodium salt

in $75 \%$ ethanol

B-naphthol

$0.1 \mathrm{gm} / 1$

1.59

in water

1-naphthylamine

$0.1 \mathrm{gm} / 1$

1.37

in $50 \%$ ethanol

$0.1 \mathrm{gm} / 1$

in $50 \%$ ethanol

Triree strong reagents (and powerful chemical quenchers in conventional scintillation mixtures) were added to the sample compartment during the tests with umbelliferone and dimethyl POPOP. One milliliter of concentrated sulfuric acid, 10\% sodiun! hydroxide, and 30\% hydrogen peroxide were each tested in separate experiments. In every case, no significant change in the relative counting efficiency was observed.

Another serjes of tests was designed to examine the effect of color quenching in the sample. Here, either the wavelength shifter dimethyl POPOP in toluene or plain water was used in the external compartment of the Cerenkov vial. Dye solutions were added to the sample compartment 
until a strong color component was visible. However, no attempt was made to determine the aciual absurbances of the solutions since relative measurements were being made. Coloring agents used were water-soluble FD\&C coal-tar dyes. The results obtained in these tests are shown in Table I?I.

Table III

THE EFFECT GF COLOR IN THE SAMPLE WHEN USING THE CERENKOV VIAL.

\begin{tabular}{|c|c|c|}
\hline Color $\& \lambda$ abs. $\max$ & Waveshifter & $\begin{array}{c}\text { Relative } \\
\text { Counting Eff. }\end{array}$ \\
\hline None & None (Water) & 1.00 \\
\hline $\begin{array}{l}\text { FD\&C yellow }(400 \mathrm{~nm}) \\
\text { FD\&C, yellow }\end{array}$ & $\begin{array}{l}\text { None } \\
\text { DitPOPOP }\end{array}$ & $\begin{array}{l}0.62 \\
0.91\end{array}$ \\
\hline $\begin{array}{l}\text { FD\&C red }(510 \mathrm{~nm}) \\
\text { FD\&C red }\end{array}$ & $\begin{array}{l}\text { Hone } \\
\text { EilPOPOP }\end{array}$ & $\begin{array}{l}0.57 \\
1.09\end{array}$ \\
\hline $\begin{array}{l}\text { FD\&C blue }\left(\begin{array}{ll}625 & \mathrm{~nm}\end{array}\right) \\
\text { FO\&C blue }\end{array}$ & $\begin{array}{l}\text { ione } \\
\text { D?OPOP }\end{array}$ & $\begin{array}{l}0.91 \\
1.83\end{array}$ \\
\hline
\end{tabular}

all tests with $5 r-89$ tracer

\section{DISCUSSION}

The results presented in Table i I clearly demonstrate that the speciaily constructed Cerenkov vial can be used to significantly increase the detection efficiency of beta emitters by separating the sample and the wavelength shiftiliy component. In the case of $5 r-89$, which is a moderately energetic emitter $\left(E_{\Gamma, a_{0}}=1.49 \mathrm{MeV}\right.$ ), a major increase is observed. Since previous work has indicated that waveshifing becomes more crucial with weaker emitters, an even greater increase could be projected for nucliues such as $\mathrm{Cl}-36$ or $\mathrm{T}_{\mathrm{m}} \mathrm{l}$ 170. A vital consideration is that use of the new vial design does not change the detection process from i true Cerenkov function. Thus, insensitivity to chemicai quenching is preserved and virtually any form of sample dissolution and pre-treatment can be employed. Another prominer:t feature of the new vial is that the wavelength shifter can be used in a totally organic system. 
This res.1ts in the ability to select more efficient fluor materials and those that matcil phototube spectral response more closely.

An additional aspect of the vial concept is the improved response when some visible color quenching renains in the sample (Table IIi). This is important because some biological materials resist complete decoloracion using simple procedures. It is only fair to note that ultraviolet absorbers in the sample will clearly reduce the effectiveness of this technique. Sut, we have found that many of these materials are easily destroyed during the routine initial sample dissolution. The only real disadvantage of this method is that the volume of sample thet. can be convenientiy handled is reduced by about a factor of two. However, in the majority of situations, sample size is not a limiting factor.

\section{REFERENCES}

1. E. H. Belcher, Proc. Roy. Soc, A216, 90 (1953).

2. J. V. Jelley, Cerenkov Radiation, New York: Pergamon Press (1958).

3. V. K. Haberer, Atomwirtschait 10, 36 (1965).

4. B. Francois, Int. J. Nuc. Med. and Biology 1, 1 (1973).

5. H. H. Ross, Ana1. Chem. 41, 1250 (1969).

6. H. H. Ross in The Current Status of Liauid Scintillation Counting, p. 123 (E. D. Bransome, Ed.). New York and London: Grune and Stratton (1970).

7. L. C. Brown, Ana). Chem. 43, 1326 (1971).

8. R. B. Randolph, Int. J. Appl. Radiation and Isotopes 26, 9 (1975).

9. K. Buchtela and M. Ischuriovits, ibid. 26, 333 (1975).

10. H. H. Ross and G. T. Rasmussen in Liquid Scintillation Counting, p. 363 (P. E. Stanley and B. A. Scoggins, Eds.). New York and London: Academic Press (1974). 
11. R. D. Smith, J. J. B. And son and M. Ristic, Int. J. App1. Radiation and Isotopes 23, 513 (1972).

12. F. Fric and V. Palovcikova, itid. 26, 305 (1975).

13. S. Seshadri, ibid. 26, 557 (1975).

14. A. Lauchli in Organic Scintillators and Liquid Scintillation Counting, p. 771 (D. L. Horrocks and C-T. Peng, Eds.). New York and London: Academic Press (1977).

15. R. H. Eirick and R. P. Parker, Int. J. Appl. Radiation and Isotopes 19, 253 (1968).

16. A. T. B. Moir, ibid. 22,213 (1971).

17. L. I. Wiebe, A. A. Noujaim and C. Edis, ibid. 22, 463 (1971).

18. J. E. Johnson and J. M. Hartsuck, Health Phys. 16, 755 (1969).

19. R. D. Stubbs and A. Jackson, Int. J. Appi. Radiation and Isotopes 18, 857 (1967).

20. P. E. Ballance and S. Johnson, Planta 91, 364 (1970!.

21. P. E. Ballance and S. Johnson, Health Phys. 20, 447 (1971).

22. H. H. Ross in Organic Scintillators and Liquid Scintillation Counting, p. 757 (D. L. Horrocks and C-T. Peng, Eds.). New York and London: Academic Press (1971).

23. E. Heiberg and J. Marshall, Rev. Sci. Instrum. 27, 618 (1956).

24. N. Porter, Nuovo Cim. 5, Series 10, 526 (1957).

25. P. Baillon, et al., Nuc. Irstrum. Methods 126,13 (i975). 
Figure 1

Quartz counting vial with isolated wavelength shifter compartment for Cerenkov counting 
ORNL-DWG. 76-8951

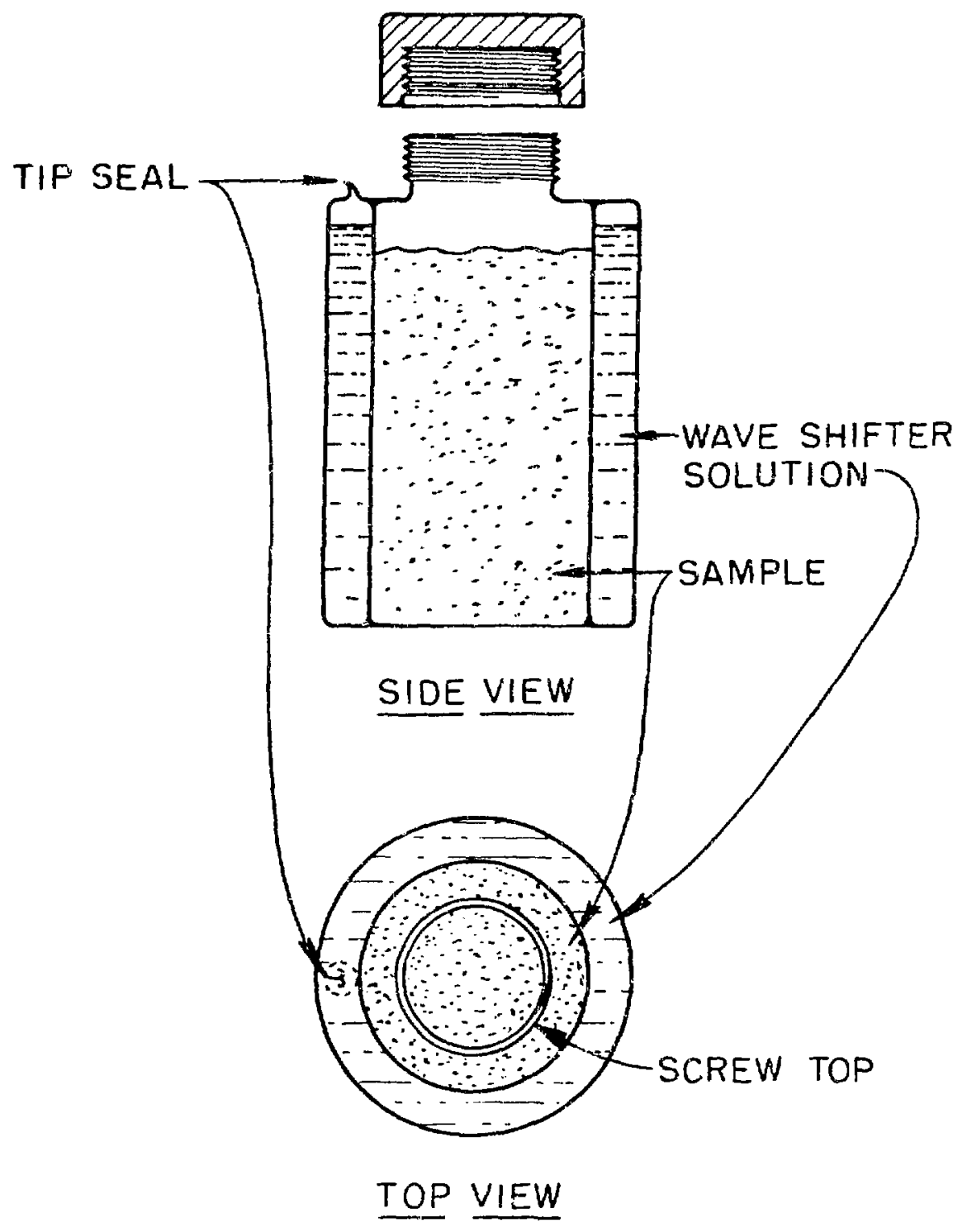

QUARTZ SAMPLE VIAL 